\title{
Astaxanthin inhibits gemcitabine-resistant human pancreatic cancer progression through EMT inhibition and gemcitabine resensitization
}

\author{
TAO YAN $^{1 *}$, HAI-YING LI ${ }^{1 *}$, JIAN-SONG WU ${ }^{1}$, QIANG NIU ${ }^{1}$, WEI-HONG DUAN ${ }^{1}$, \\ QING-ZENG HAN ${ }^{2}$, WANG-MING JI ${ }^{1}$, TAO ZHANG ${ }^{1}$ and WEI LV ${ }^{1}$ \\ ${ }^{1}$ Department of Hepatobiliary Surgery, The General Hospital of The PLA Rocket Force, Beijing 100088; \\ ${ }^{2}$ Surgical Department, Qinghe County Central Hospital, Qinghe, Xingtai, Hebei 054800, P.R. China
}

Received January 9, 2016; Accepted June 16, 2017

DOI: $10.3892 / 01.2017 .6836$

\begin{abstract}
Pancreatic cancer rapidly acquires resistance to chemotherapy resulting in its being difficult to treat. Gemcitabine is the current clinical chemotherapy strategy; however, owing to gemcitabine resistance, it is only able to prolong the life of patients with pancreatic cancer for a limited number of months. Understanding the underlying molecular mechanisms of gemcitabine resistance and selecting a suitable combination of agents for the treatment of pancreatic cancer is required. Astaxanthin (ASX) is able to resensitize gemcitabine-resistant human pancreatic cancer cells (GR-HPCCs) to gemcitabine. ASX was identified to upregulate human equilibrative nucleoside transporter 1 (hENT1) and downregulate ribonucleoside diphosphate reductase (RRM) 1 and 2 to enhance gemcitabine-induced cell death in GR-HPCCs treated with gemcitabine, and also downregulates TWIST1 and ZEB1 to inhibit the gemcitabine-induced epithelial-mesenchymal transition (EMT) phenotype in GR-HPCCs and to mediate hENT1, RRM1 and RRM2. Furthermore, ASX acts through the hypoxia-inducible factor $1 \alpha /$ signal transducer and activator of transcription 3 signaling pathway to mediate TWIST1, ZEB1, hENT1, RRM1 and RRM2, regulating the gemcitabine-induced EMT phenotype and gemcitabine-induced cell death. Co-treatment with
\end{abstract}

Correspondence to: Dr Wei Lv, Department of Hepatobiliary Surgery, The General Hospital of The PLA Rocket Force, 16 Xinjie Kouwai Street, Beijing 100088, P.R. China

E-mail: weil_1968@sina.com

*Contributed equally

Abbreviations: ASX, astaxanthin; EMT, epithelial-to-mesenchymal transition; GR-HPCCs, gemcitabine-resistant human pancreatic cancer cells; HPCCs, human pancreatic cancer cells; hENT1, human equilibrative nucleoside transporter 1; RRM, ribonucleoside diphosphate reductase

Key words: astaxanthin, gemcitabine, epithelial-mesenchymal transition, gemcitabine-resistance human pancreatic cancer cells
ASX and gemcitabine in a tumor xenograft model induced by GR-HPCCs supported the in vitro results. The results of the present study provide a novel therapeutic strategy for the treatment of gemcitabine-resistant pancreatic cancer.

\section{Introduction}

Pancreatic cancer is a lethal malignancy, with a mortality rate of $>90 \%$; it is ranked fourth in terms of cancer-related mortality (1-3). Although the treatment of pancreatic cancer involves chemotherapy, patients typically receive treatment in the terminal stage, owing to a lack of effective detection methods for the early-stage disease (3). Gemcitabine is the chemotherapeutic agent used for the treatment of pancreatic cancer, and the effect of gemcitabine is marked, with suppression of tumor growth, leading to the prolonging of patient survival, but only for a limited number of months (4). In order to enhance the effect of gemcitabine, studies have focused on combination chemotherapy, but only a limited number of agents have been screened, therefore a novel combination chemotherapy agent is required. Owing to the rapid development of gemcitabine resistance in pancreatic cancer, the effect of gemcitabine is limited. The aim of the present study was to investigate the underlying molecular mechanisms of gemcitabine resistance, and identify a suitable target combination chemotherapy agent that may promote the effect of gemcitabine for the treatment of pancreatic cancer.

Human equilibrative nucleoside transporter 1 (hENT1) is able to carry pyrimidine nucleosides and purine into cells (5). Previous studies have identified that hENT1 serves an important role in gemcitabine resistance $(6,7)$. Patients with pancreatic cancer with increased hENT1 expression levels have an increased lifespan compared with those with lower hENT1 expression under gemcitabine treatment (8). These results suggest that hENT1 may be a target of gemcitabine resistance in pancreatic cancer, therefore hENT1 agonist combination chemotherapy may promote the effect of gemcitabine. Furthermore, ribonucleoside diphosphate reductase (RRM) 1 and 2 are target molecules of gemcitabine, as, when RRM1 and RRM2 expression levels are low, patients with pancreatic cancer exhibit 
increased sensitivity to gemcitabine chemotherapy (9). Previous studies have suggested that RRM1 and RRM2 expression levels are increased in patients with gemcitabine resistance (10-12). Therefore, selecting suitable RRM1 and RRM2 target agents may be a means to overcome gemcitabine resistance.

Epithelial-mesenchymal transition (EMT) results in the loss of cell-cell junctions and migratory and invasive mesenchymal cell formation (13). Transforming growth factor- $\beta$ and Notch signaling pathways have been demonstrated to mediate the gemcitabine-resistance-induced EMT (13). Associations between EMT and cancer aggressiveness have been demonstrated in pancreatic cancer, and certain studies have suggested an association between EMT and gemcitabine resistance in pancreatic cancer $(14,15)$. Therefore, preventing gemcitabine-resistance-induced EMT may be a novel therapeutic strategy.

Astaxanthin (ASX) is a lipophilic compound, exhibiting antioxidant, anti-inflammatory and immunomodulatory characteristics. The anticancer ability of antioxidants has been a focus of research, particularly the effect of oxidative stress and metabolism. Powerful antioxidants may be novel and effective agents for the treatment of carcinoma. Previous studies have demonstrated that ASX was able to inhibit the proliferation of various types of cancer cell through immunomodulatory and cell communication modulation at gap junctions $(16,17)$. However, there has been limited research on ASX acting as a combination chemotherapy agent with gemcitabine in the treatment of human pancreatic cancer. The aim of the present study was to investigate whether ASX was able to promote the effect of gemcitabine, and suppress gemcitabine resistance and promote gemcitabine-induced cell death in human pancreatic cancer cells (HPCCs), namely Panc-1 and HTB-79. ASX was identified to promote hENT1 expression levels and inhibit gemcitabine-resistance-induced EMT through the hypoxia-inducible factor $1 \alpha(\mathrm{HIF}-1 \alpha)$ /signal transducer and activator of transcription 3 (STAT3)-TWIST1/ZEB1 signaling pathway in pancreatic cancer cells. Furthermore, ASX in combination with gemcitabine was able to significantly suppress tumor growth in a gemcitabine-resistant pancreatic cancer cell-induced pancreatic tumor model. Therefore, ASX and gemcitabine co-treatment provides a novel combination chemotherapy strategy for targeting gemcitabine-resistant pancreatic cancer.

\section{Materials and methods}

Cell culture. The HPCCs Panc-1 and HTB-79 were purchased from the American Type Culture Collection (Manassas, VA, USA). All cells were cultured in Dulbecco's modified Eagle's medium containing $10 \%$ fetal bovine serum (FBS) and $100 \mathrm{U} / \mathrm{ml}$ penicillin, at $37^{\circ} \mathrm{C}$ and $5 \% \mathrm{CO}_{2}$. All cell culture regents were purchased from Gibco; Thermo Fisher Scientific, Inc. (Waltham, MA, USA). Panc-1 and HTB-79 cells were exposed to gemcitabine at increasing concentrations from $10 \mathrm{nM}$ to generate gemcitabine-resistant cells. After 2 weeks of adaptation, the concentration was doubled. The final gemcitabine concentration to which the cells were adapted was $640 \mathrm{nM}$, and the cells were designated GR-Panc-1 cells and GR-HTB-79 cells.
Western blot analysis. For each sample, Panc-1 and HTB-79 cells were lysed for $30 \mathrm{~min}$ in radioimmunoprecipitation assay lysis buffer (Beyotime Institute of Biotechnology, Shanghai, China) on ice, and the cell debris was centrifuged at $12,000 \mathrm{x} \mathrm{g}$ for $8 \mathrm{~min}$ at $4^{\circ} \mathrm{C}$. The protein concentration was determined using the bicinchoninic acid assay, and $40 \mu \mathrm{g}$ proteins were separated by SDS-PAGE (10-15\% gels) and blotted onto a polyvinylidene fluoride (PVDF) membrane. The PVDF membrane was blocked in a solution of 5\% non-fat dried milk in PBST $(0.05 \%$ Tween-20 in PBS) for $1 \mathrm{~h}$ at room temperature, and incubated with antibodies at $4^{\circ} \mathrm{C}$ for $12 \mathrm{~h}$. The PVDF membrane was washed three times for $10 \mathrm{~min}$ in PBST, incubated with secondary antibodies at $37^{\circ} \mathrm{C}$ for $1 \mathrm{~h}$, and washed again three times for $10 \mathrm{~min}$ in PBST. GAPDH was used as a loading control. All western blotting reagents were purchased from Beyotime Institute of Biotechnology. All the antibodies were purchased from Santa Cruz Biotechnology, Inc. (Dallas, TX, USA). The first antibodies were diluted at 1:500, and the secondary antibodies were diluted at 1:5,000. The following antibodies were used: hENT1 (cat. no. sc-48489; polyclonal, goat anti-human), RRM1 (cat. no. sc-22786; monoclonal, rabbit anti-human), RRM2 (cat. no. sc-137174; monoclonal, mouse anti-human), GAPDH (cat. no. sc-293335; monoclonal, mouse anti-human), Twist1 (cat. no. sc-134136, polyclonal, mouse anti-human), ZEB1 (cat. no. sc-517272; monoclonal, mouse anti-human), E-cadherin (cat. no. sc-33743; polyclonal, rabbit anti-human), STAT3 (cat. no. sc-8059; monoclonal, mouse anti-human), $\alpha$-SMA (cat. no. sc-53142; monoclonal, mouse anti-human), HIF-1 $\alpha$ (cat. no. sc-13515; monoclonal, mouse anti-human), mouse IgG (cat. no. sc-516176), rabbit IgG (cat. no. sc-2794) and goat IgG (cat. no. sc-2419).

Cell viability assay. Each well of a 96-well plate was inoculated with $10^{4}$ Panc-1 or HTB-79 cells and cells were allowed to attach overnight, prior to treatment with drugs for $24 \mathrm{~h}$. The medium was removed and the cells were washed three times with PBS, prior to the addition of $90 \mu$ l Dulbecco's modified Eagle's medium (DMEM) and $10 \mu \mathrm{l}$ Cell Counting kit-8 reagent (Beyotime Institute of Biotechnology) to each well. Cells were incubated at $37^{\circ} \mathrm{C}$ for $1 \mathrm{~h}$ before determining the optical density at $450 \mathrm{~nm}$ using a microplate reader.

Trypan blue assay. Treated Panc-1 or HTB-79 cells suspensions were obtained and a $1 / 9$ volume of $0.4 \%(w / v)$ trypan blue solution was added. The number of total cells and dead cells (those that did not exclude the dye) were determined, and the total death rate was calculated as (number of dead cells/number of total cells) x100\%.

Transfection experiment. Transient transfection of GR-Panc-1 cells with small interfering RNAs (siRNAs) and plasmids was performed using Lipofectamine 2000 (Invitrogen; Thermo Fisher Scientific, Inc.), the siRNAs were purchased from Shanghai Genechem Co., Ltd. (Shanghai, China), and the sequences of siRNAs were as follows: Control, 5'-UUC UCCGAACGUGUCACGUTT-3'; hENT1, 5'-AUGACAUUG UUGAAGAUGGCA-3'; ZEB1, 5'-GGACUGAAGUCAGGU AAGGCA-3'; Twist1, 5'-AAACAUUUGUUUUAAGGA GAA-3'; STAT3, 5'-GCUAAGUCAGCUUCAUUGAGU-3'; and HIF-1 $\alpha, 5^{\prime}$-GCAUUGCCAUCAGUCACGCUA-3'. The 
con-pcDNA3.1, RRM1-pcDNA3.1 and RRM2-pcDNA3.1 plasmids were purchased from Shanghai Genechem Co., Ltd. The 6-wells plates were used for siRNA or plasmid transfection assays. For the each well, $500 \mathrm{ng}$ siRNAs or $2 \mu \mathrm{g}$ plasmids were added to $10 \mu \mathrm{l}$ Lipofectamine 2000 according to the manufacturer's protocol, and were incubated with GR-Panc-1 cells for $48 \mathrm{~h}$. The siRNAs or plasmids were then removed, and GR-Panc-1 cells were treated with drugs for an additional $24 \mathrm{~h}$. Subsequently, the cells were collected for western blot analysis, as aforementioned.

Cell invasion assay. A BioCoat Matrigel invasion chamber system (Corning Incorporated, Corning, NY, USA) was used to assay cell invasion. Using a Transwell plate, the lower chamber was filled with culture medium without cells, and the upper chamber was filled with cell suspension and medium containing 10\% FBS. The Transwell plate was incubated at $37^{\circ} \mathrm{C}$ for $24 \mathrm{~h}$. Cells that adhered to the upper chamber surface were removed, and the cells that adhered to the lower chamber surface were stained with $4 \%$ paraformaldehyde for $15 \mathrm{~min}$, rinsed with water and dried. The $0.5 \%$ crystal violet was extracted with $50 \%$ ethanol containing $0.1 \mathrm{M}$ sodium citrate, and the absorbance at $600 \mathrm{~nm}$ was determined.

Xenograft model. A total of 30 male 6-week-old BALB/c nude mice (weighing 18-22 g) were purchased from the Institute of Zoology (Chinese Academy of Sciences, Beijing, China). All animal experiments were performed according to the guidelines of the Institutional Animal Care and Use Committee of Institute of Zoology (Chinese Academy of Sciences, Beijing, China). All the animals were fed in a pathogen-free environment, at $24-28^{\circ} \mathrm{C}$. Ventilation was required $10-20$ times per hour, relative humidity was $50-60 \%$, the light/dark cycle was natural circadian light, the food was sterilized by irradiation, and the water contained bacitracin $(4 \mathrm{~g} / \mathrm{l})$ and neomycin $(4 \mathrm{~g} / \mathrm{l})$. Each BALB/c nude mouse was subcutaneously inoculated with $5 \times 10^{6}$ Panc-1 or GR-Panc-1 cells into the right and left hind footpads. At 2 days after inoculation, the mice inoculated with Panc-1 cells were treated with $10 \mathrm{mg} / \mathrm{kg}$ gemcitabine 3 times/day by intraperitoneal injection, and the mice inoculated with GR-Panc-1 cells were treated with $10 \mathrm{mg} / \mathrm{kg}$ gemcitabine or co-treated with $500 \mathrm{mg} / \mathrm{kg}$ ASX and $10 \mathrm{mg} / \mathrm{kg}$ gemcitabine 3 times/day by intraperitoneal injection, with the injection of ASX occurring $2 \mathrm{~h}$ before that of gemcitabine. ASX and gemcitabine were dissolved in saline. Tumor volumes were determined weekly.

Terminal deoxynucleotidyltransferase-mediated dUTP nick-end labeling (TUNEL) assay. Tumors were immersed in $4 \%$ paraformaldehyde for $24 \mathrm{~h}$ and dehydrated in $30 \%$ sucrose solutions, prior to paraffin-embedding and cutting into sections $(10 \mu \mathrm{m})$. The sections were treated using an In Situ Cell Death Detection kit (Roche Diagnostics, Indianapolis, IN, USA), according to the manufacturer's protocol.

Statistical analysis. Results are presented as the mean \pm standard deviation of triplicate experiments and were performed with SPSS 17.0 (SPSS, Inc., Chicago, IL, USA). Two-way analysis of variance, followed by Bonferroni post hoc testing, was used to compare different groups. The unpaired Student t-test or Mann-Whitney U test was used to compare two means. $\mathrm{P}<0.05$ was considered to indicate a statistically significant difference.

\section{Results}

ASX enhances gemcitabine-induced pancreatic cancer cell death. Using increasing gemcitabine concentrations starting at $10 \mathrm{nM}$ to induce Panc-1 and HTB-79 GR-HPCCs, designated GR-Panc-1 and GR-HTB-79. Panc-1 and HTB-79 cells were treated with various concentrations of gemcitabine; cell viability was identified to decrease with increasing gemcitabine concentration, and the cell death ratio was identified to increase with increasing gemcitabine concentration (Fig. 1A). In contrast, GR-HPCCs were treated with the same concentrations of gemcitabine, but no alteration in cell viability or cell death ratio was observed with increasing gemcitabine concentration (Fig. 1A). When GR-HPCCs were treated with various concentrations of gemcitabine in combination with $200 \mu \mathrm{M}$ ASX, the cell viability and cell death ratio were significantly different compared with treatment with gemcitabine alone, which occurred in a gemcitabine dose-dependent manner (Fig. 1B). When GR-HPCCs were treated with various concentrations of ASX in combination with $1 \mu \mathrm{M}$ gemcitabine, the cell viability and cell death ratio were significantly different compared with treatment with ASX alone, which occurred in an ASX dose-dependent manner (Fig. 1C). These results indicated that ASX enhances gemcitabine-induced GR-HPCC death.

ASX upregulates $h E N T 1$ and downregulates RRM1 and RRM2 expression. It has been demonstrated previously that hENT1, RRM1 and RRM2 serve important roles in the anticancer efficiency of gemcitabine $(6,18,19)$. RRM1 and RRM2 are targets of gemcitabine: When RRM1 and RRM2 expression levels are increased, patients exhibit only limited sensitivity to gemcitabine chemotherapy, appearing gemcitabine-resistant. Determining the expression level of hENT1, RRM1 and RRM2 in HPCCs and GR-HPCCs treated with gemcitabine or gemcitabine in combination with ASX, it was identified that the hENT1 expression level in GR-HPCCs was decreased compared with that in HPCCs, and the RRM1 and RRM2 expression level in GR-HPCCs was increased compared with that in HPCCs, ASX was able to enhance the hENT1 expression level and inhibit the RRM1 and RRM2 expression level in GR-HPCCs (Fig. 2A). By knocking down hENT1 using siRNA in GR-Panc-1 cells, it was identified that the cell death ratio was decreased compared with cells transfected with Con-siRNA, including cells co-treated with gemcitabine and ASX (Fig. 2B). RRM1 and RRM2 overexpression was also able to inhibit the gemcitabine- and ASX-induced cell death ratio in GR-Panc-1 cells (Fig. 2C and D). These results indicate that ASX may enhance gemcitabine-induced GR-HPCC death by targeting hENT1 and RRM1 and RRM2.

ASX suppresses the EMT phenotype in GR-HPCCs. To investigate whether ASX affects the EMT phenotype which was established to investigate gemcitabine resistance, the expression epithelial (E-)cadherin, TWIST1, ZEB1 and $\alpha$-smooth muscle actin ( $\alpha$-SMA) were examined in HPCCs 

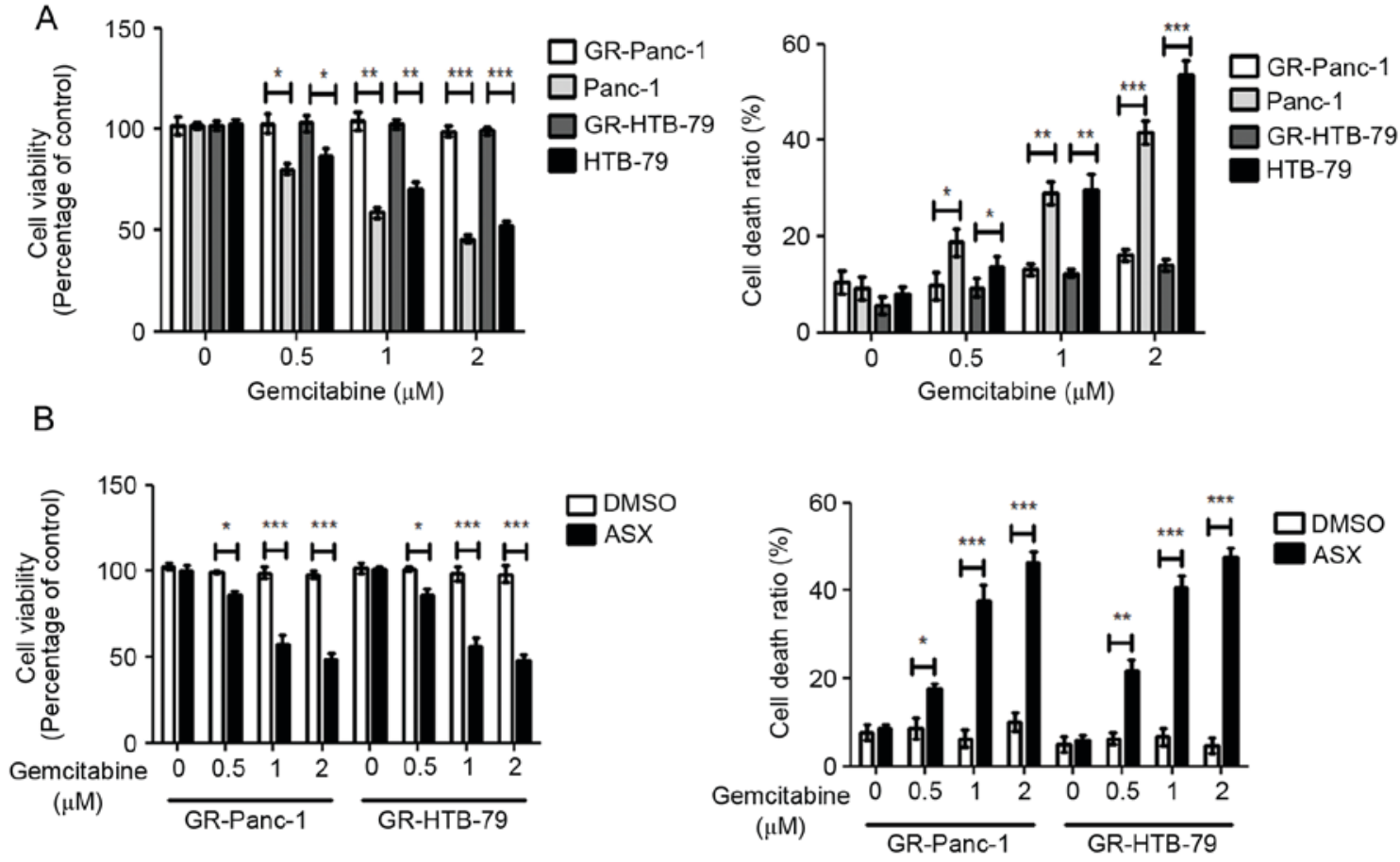

C
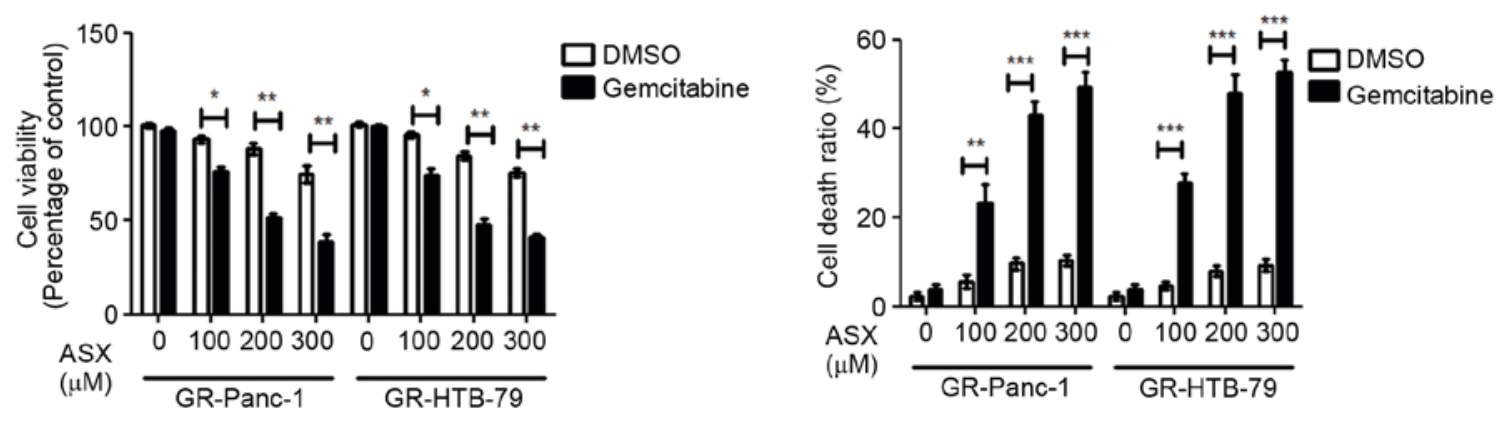

Figure 1. ASX enhances gemcitabine-induced pancreatic cancer cell death. (A) HPCCs and GR-HPCCs were treated with various concentrations of gemcitabine for $24 \mathrm{~h}$. (B) GR-HPCCs were pretreated with $0.1 \%$ DMSO or $200 \mu \mathrm{M}$ ASX for $2 \mathrm{~h}$, and then treated with various concentrations of gemcitabine for $24 \mathrm{~h}$. (C) GR-HPCCs were pretreated with various concentrations of ASX for $2 \mathrm{~h}$, and then treated with $1 \mu \mathrm{M}$ gemcitabine or $0.1 \%$ DMSO for $24 \mathrm{~h}$. Cell viability was determined using the Cell Counting kit- 8 . The cell death ratio was determined using a trypan blue assay. ${ }^{*} \mathrm{P}<0.05 ;{ }^{* * *} \mathrm{P}<0.01$; ${ }^{* * *} \mathrm{P}<0.005$. ASX, astaxanthin; HPCCs, human pancreatic cancer cells; GR-HPCCs, gemcitabine-resistant HPCCs; DMSO, dimethyl sulfoxide.

and GR-HPCCs treated with gemcitabine or co-treated with gemcitabine and ASX. It was identified that the expression levels of the mesenchymal cell markers in GR-HPCCs were increased compared with those in HPCCs, and that of the epithelial cell marker was decreased (Fig. 3A). Furthermore, ASX was able to decrease mesenchymal cell marker expression and increase epithelial cell marker expression in GR-HPCCs treated with gemcitabine (Fig. 3A). TWIST1 and ZEB1 are transcription factors that are able to mediate EMT, and are overexpressed in a number of cancer EMT phenotypes $(20,21)$. Thus, it was hypothesized TWIST1 and ZEB1 were the key factors in the ASX-mediated EMT phenotype in GR-HPCCs. In order to investigate this hypothesis, TWIST1 was knocked down through siRNA transfection in GR-Panc-1 cells, and the EMT phenotype was inhibited by decreasing $\alpha$-SMA and increasing E-cadherin expression, and hENT1 expression was increased. In addition, expression of RRM1 and RRM2 was inhibited in GR-Panc-1 cells with TWIST1 knocked down (Fig. 3B). Furthermore, the cell death ratio was detected, and the results showed that the cell death ratio was enhanced when TWIST1 was knocked down in GR-Panc-1 cells (Fig. 3C). Similarly, ZEB1 was knocked down in GR-Panc-1 cells, the EMT and cell death ratio were detected, and the results indicated that the EMT was inhibited (Fig. 3D). When ZEB1 was knocked down through ZEB1-siRNA transfection, the expression of hENT1 was increased while RRM1 and RRM2 expression was inhibited (Fig. 3D). In addition, the cell death ratio also was improved in GR-Panc-1 cells when ZEB1 was knocked down (Fig. 3E). Cell invasion assays indicated that the invasion of GR-HPCCs was increased compared with that of HPCCs, and ASX was able to inhibit GR-HPCC invasion (Fig. 3F). These results suggested that ASX is able to suppress the EMT phenotype and enhance GR-HPCC sensitization to gemcitabine through the TWIST1 and ZEB1 signaling pathway.

ASX resensitizes GR-HPCCs to gemcitabine-induced cell death through the HIF-1 //STAT3 signaling pathway. It has been demonstrated previously that HIF- $1 \alpha$ and STAT3 were mediators of TWIST1 and ZEB1 (22-24). The aforementioned 

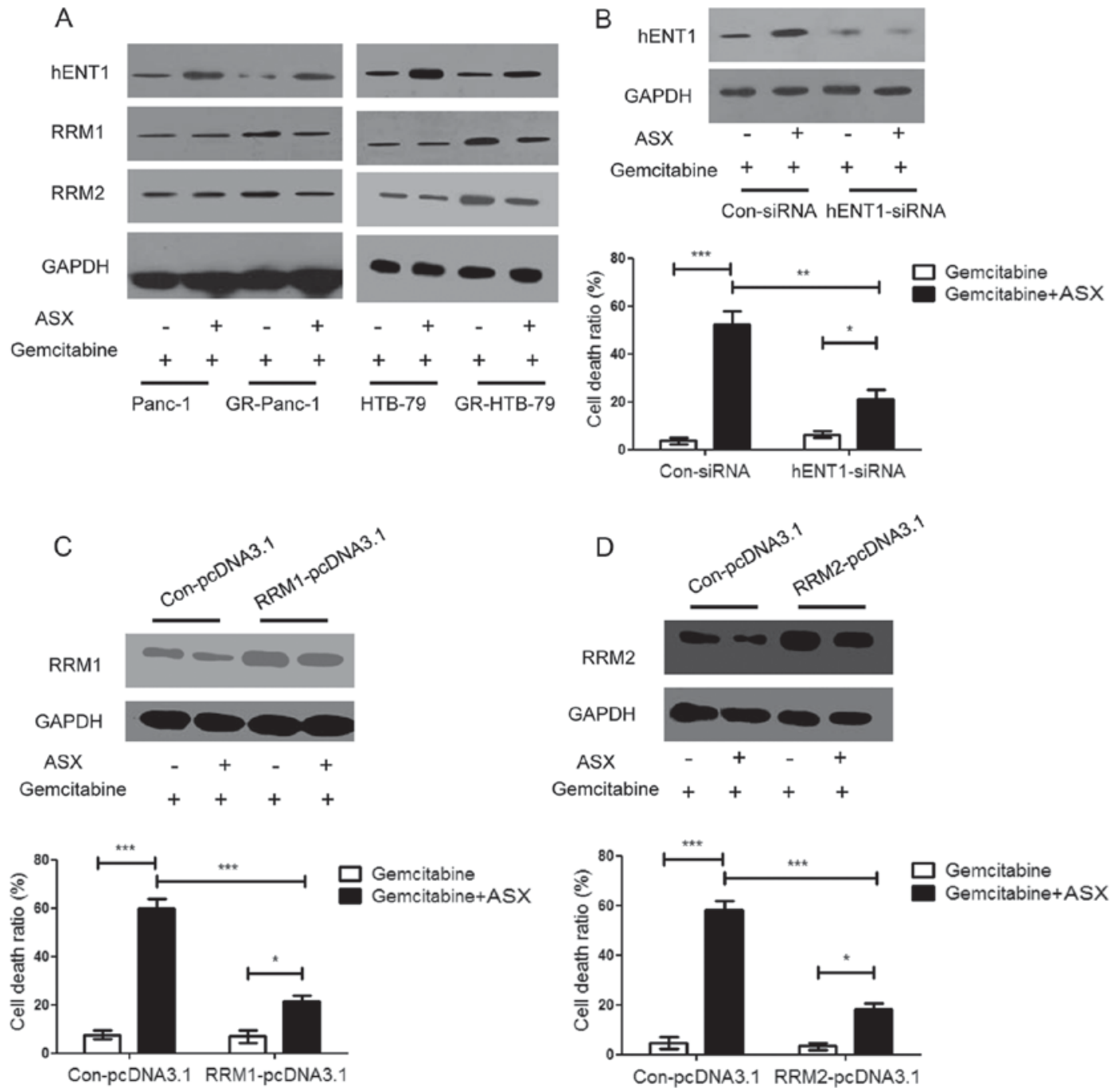

Figure 2. ASX upregulates hENT1 and downregulates RRM1 and RRM2 expression in GR-HPCCs, and increases gemcitabine-induced GR-HPCC death through hENT1, RRM1 and RRM2 signaling. (A) HPCCs and GR-HPCCs were treated with $1 \mu \mathrm{M}$ gemcitabine alone or co-treated with $200 \mu \mathrm{M}$ ASX $(2 \mathrm{~h}$ pretreatment) and $1 \mu \mathrm{M}$ gemcitabine for $24 \mathrm{~h}$. ASX was able to restore the decrease in hENT1 protein expression and restore the RRM1 and RRM2 protein expression levels in GR-HPCCs. (B) GR-Panc-1 cells were transfected with control siRNA or hENT1-siRNA, and treated with $1 \mu \mathrm{M}$ gemcitabine alone or co-treated with $200 \mu \mathrm{M}$ ASX ( $2 \mathrm{~h}$ pretreatment) and $1 \mu \mathrm{M}$ gemcitabine for $24 \mathrm{~h}$. The cell death ratio was analyzed using a trypan blue assay. GR-Panc-1 cells were transfected with (C) RRM1 plasmid (RRM1-pcDNA3.1) or (D) RRM2 plasmid (RRM2-pcDNA3.1) or control plasmid (Con-pcDNA3.1), and treated with $1 \mu \mathrm{M}$ gemcitabine alone or co-treated with $200 \mu \mathrm{M}$ ASX ( $2 \mathrm{~h}$ pretreatment) and $1 \mu \mathrm{M}$ gemcitabine for $24 \mathrm{~h}$. The cell death ratio was determined using a trypan blue assay. "P $<0.05 ;{ }^{* *} \mathrm{P}<0.01 ;{ }^{* * * *} \mathrm{P}<0.005$. ASX, astaxanthin; hENT1, human equilibrative nucleoside transporter 1; RRM, ribonucleoside diphosphate reductase; GR-HPCCs, gemcitabine-resistant HPCCs; HPCCs, human pancreatic cancer cells; DMSO, dimethyl sulfoxide.

results indicated that ASX is able to suppress the EMT phenotype and enhance GR-HPCC sensitization to gemcitabine through the TWIST1 and ZEB1 signaling pathway. In order to investigate the role of factors upstream of TWIST1 and ZEB1, HIF-1 $\alpha$ and STAT3 expression levels were determined in HPCCs and GR-HPCCs, which were treated with gemcitabine or co-treated with gemcitabine and ASX. It was identified that HIF-1 $\alpha$ and STAT3 expression levels in GR-HPCCs were increased compared with those in HPCCs (Fig. 4A). Furthermore, ASX was able to inhibit HIF-1 $\alpha$ and STAT3 expression (Fig. 4A). Knocking down STAT3 expression through siRNA transfection could lead to TWIST1, ZEB1, RRM1 and RRM2 expression inhibition, and an increase in hENT1 expression in GR-Panc-1 cells, and ASX could regulate the expression levels for the aforementioned molecules (Fig. 4B). In addition, when STAT3 was knocked down in GR-Panc-1 cells, ASX could effectively improve the gemcitabine-induced cell death ratio (Fig. 4C). At the same time, HIF-1 $\alpha$ was also knocked down through siRNA transfection in GR-Panc-1 cells, and western blot analysis and trypan blue stain assays were performed on the treated cells. The results suggested that TWIST1, ZEB1, RRM1 and RRM2 expression levels were inhibited and hENT1 expression level was improved when HIF-1 $\alpha$ was knocked down in GR-Panc-1 cells, and ASX also could regulate the expression levels for the aforementioned molecules (Fig. 4D). In addition, in GR-Panc-1 cells, when HIF-1 $\alpha$ was knocked down, ASX could effectively improve the gemcitabine-induced cell death ratio (Fig. 4E). These results indicated that ASX resensitizes GR-HPCCs to gemcitabine-induced cell death through the HIF-1 $\alpha /$ STAT3 signaling pathway.

ASX inhibits the growth of gemcitabine-resistant pancreatic tumors in vivo through apoptosis. The aforementioned results 
A

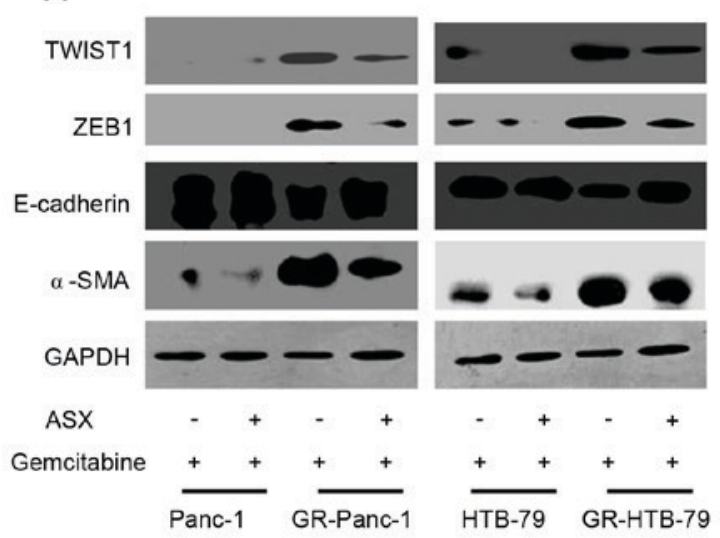

B

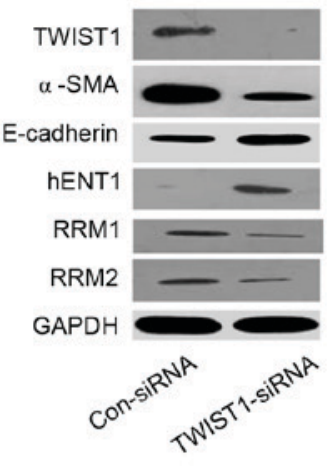

C

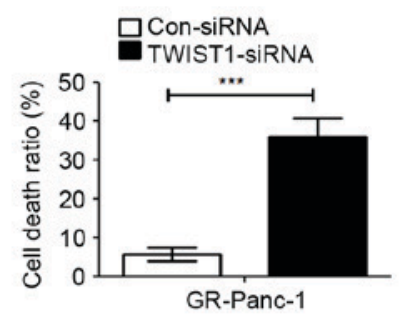

D

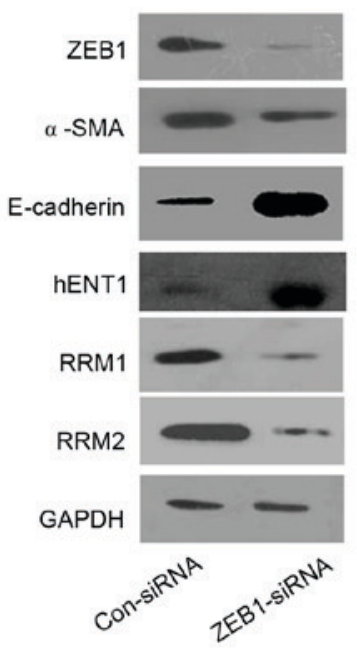

E
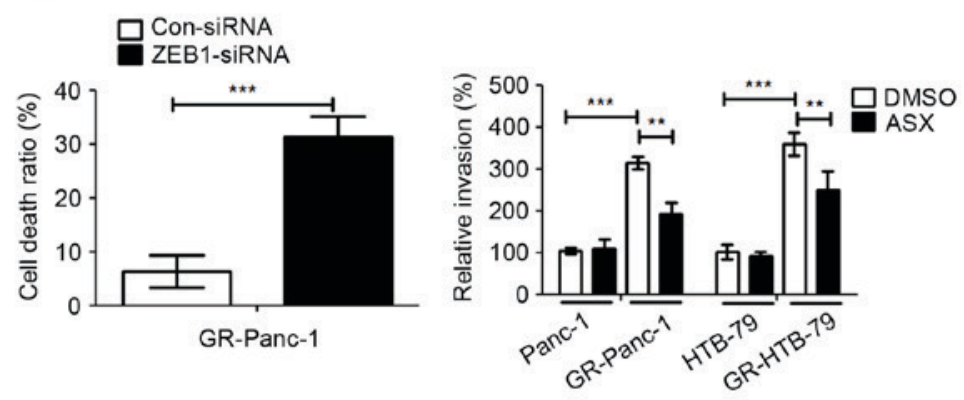

$\mathrm{F}$

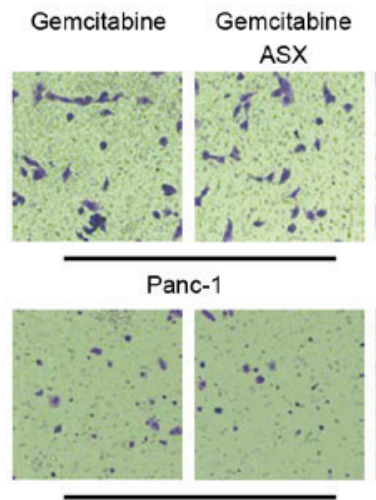

HTB-79
Gemcitabine Gemcitabine

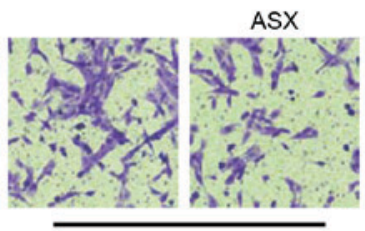

GR-Panc-1

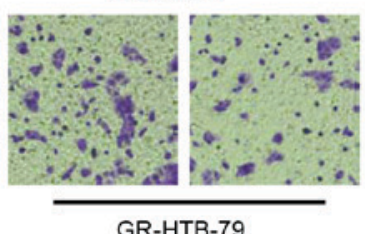

Figure 3. ASX suppresses the EMT phenotype of GR-HPCCs through TWIST1 and ZEB1 signaling, and recover the activation of TWIST1 and ZEB1 in GR-HPCCs. (A) HPCCs and GR-HPCCs were treated with $1 \mu \mathrm{M}$ gemcitabine alone or co-treated with $200 \mu \mathrm{M}$ ASX (2 h pretreatment) and $1 \mu \mathrm{M}$ gemcitabine for $24 \mathrm{~h}$. (B) GR-Panc-1 cells were transfected with control siRNA (Con-siRNA) or TWIST1-siRNA. (C) The cell death ratio was determined using a trypan blue assay. (D) GR-Panc-1 cells were transfected with control siRNA (Con-siRNA) or ZEB1-siRNA. (E) The cell death ratio was determined using a trypan blue assay. (F) ASX suppresses the invasive ability of GR-HPCCs. HPCCs and GR-HPCCs were treated with $1 \mu \mathrm{M}$ gemcitabine alone or co-treated with $200 \mu \mathrm{M}$ ASX (2 h pretreatment) and $1 \mu \mathrm{M}$ gemcitabine for $24 \mathrm{~h} .{ }^{* *} \mathrm{P}<0.01 ;{ }^{* * *} \mathrm{P}<0.005$. ASX, astaxanthin; EMT, epithelial-mesenchymal transition; GR-HPCCs, gemcitabine-resistant HPCCs; HPCCs, human pancreatic cancer cells; siRNA, small interfering RNA; E-cadherin, endothelial cadherin; $\alpha$-SMA, $\alpha$-smooth muscle actin; hENT1, human equilibrative nucleoside transporter 1; RRM, ribonucleoside diphosphate reductase.

indicated that ASX is able to stimulate gemcitabine-induced cell death in GR-HPCCs in vitro. In order to confirm this effect in vivo, a pancreatic tumor xenograft model was used. Nude mice were xenografted with Panc-1 or GR-Panc-1 cells. As expected, in the presence of gemcitabine, the tumor volume induced by Panc-1 cells was significantly decreased compared with that induced by GR-Panc-1 cells (Fig. 5A). For the tumor induced by GR-Panc-1, co-treatment with ASX and gemcitabine led to a significant decrease in the tumor volume compared with treatment with gemcitabine alone (Fig. 5A). In order to investigate the reason for the decrease in tumor volume, TUNEL was used to detect apoptosis, with the results indicating that gemcitabine was able to induce apoptosis in the xenografted Panc-1 model, and that co-treatment with ASX and gemcitabine was able to induce apoptosis in the xenografted GR-Panc-1 model (Fig. 5B and C).

\section{Discussion}

In the present study, it was identified that ASX is able to selective kill GR-HPCCs by increasing sensitivity to gemcitabine. The results of the present study indicated that GR-HPCCs 
A

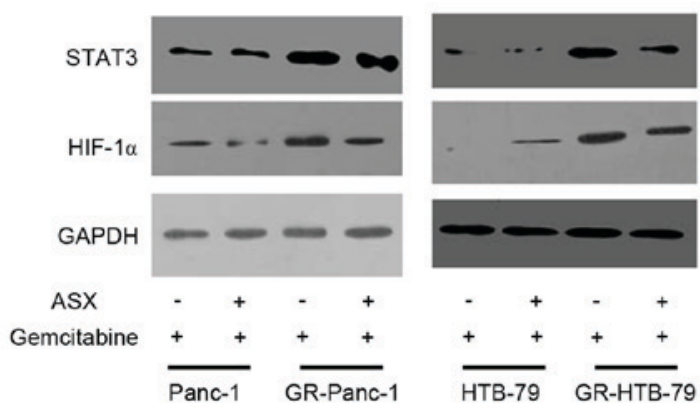

B

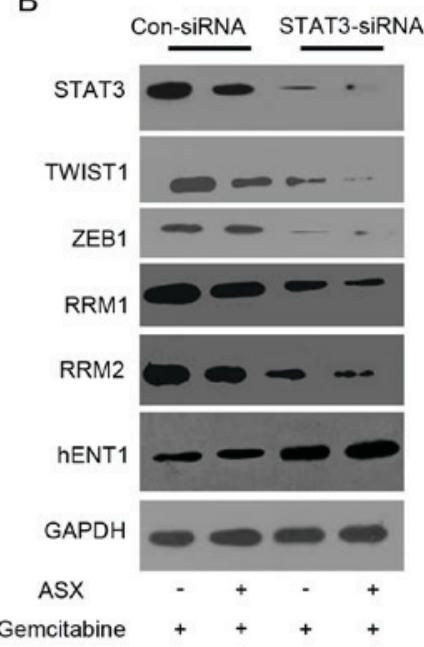

C
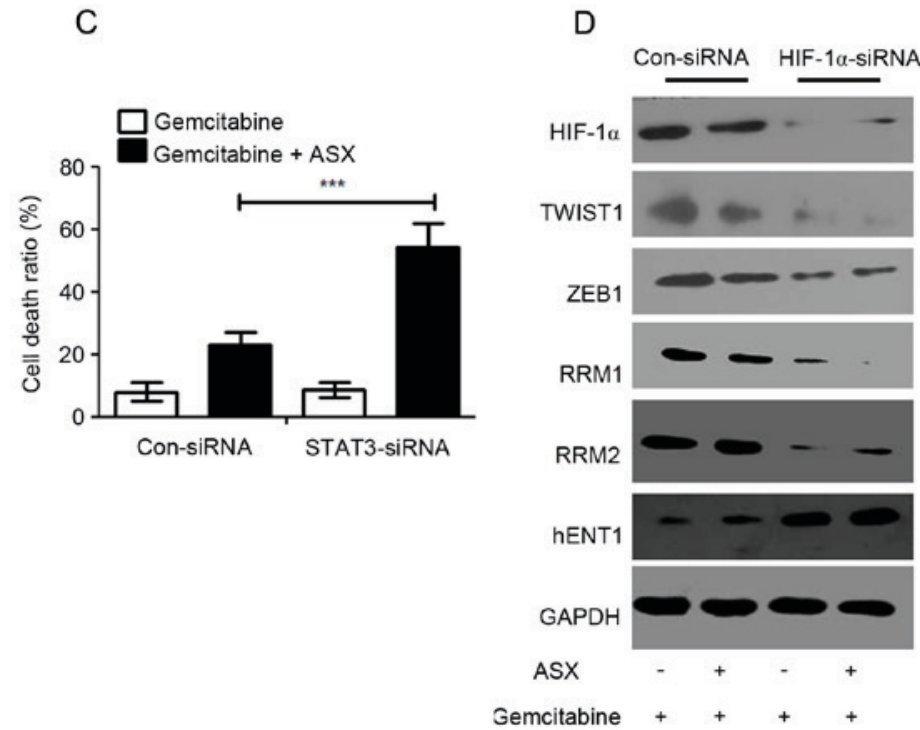

E

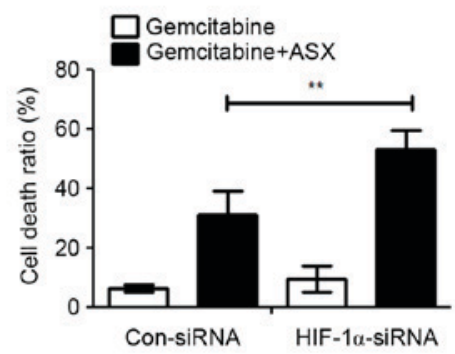

Figure 4. ASX resensitizes GR-HPCCs to gemcitabine-induced cell death through the HIF-1 $\alpha$ /STAT3 signaling pathway. (A) HPCCs and GR-HPCCs were treated with $1 \mu \mathrm{M}$ gemcitabine alone or co-treated with $200 \mu \mathrm{M}$ ASX ( $2 \mathrm{~h}$ pretreatment) and $1 \mu \mathrm{M}$ gemcitabine for $24 \mathrm{~h}$. (B) GR-Panc-1 was transfected with control siRNA (Con-siRNA) or STAT3-siRNA, and treated with $1 \mu \mathrm{M}$ gemcitabine alone or co-treated with $200 \mu \mathrm{M}$ ASX $(2 \mathrm{~h}$ pretreatment) and $1 \mu \mathrm{M}$ gemcitabine for $24 \mathrm{~h}$. (C) The cell death ratio was determined using a trypan blue assay. (D) GR-Panc-1 was transfected with control siRNA (Con-siRNA) or HIF-1 $\alpha$-siRNA, and treated with $1 \mu \mathrm{M}$ gemcitabine alone or co-treated with $200 \mu \mathrm{M}$ ASX ( $2 \mathrm{~h}$ pretreatment) and $1 \mu \mathrm{M}$ gemcitabine for $24 \mathrm{~h}$. (E) The cell death ratio was determined using a trypan blue assay. ${ }^{* *} \mathrm{P}<0.01 ;{ }^{* *} \mathrm{P}<0.01$. ASX, astaxanthin; GR-HPCCs, gemcitabine-resistant HPCCs; HIF-1 $\alpha$, hypoxia-inducible factor $1 \alpha$; HPCCs, human pancreatic cancer cells; siRNA, small interfering RNA; STAT3, signal transducer and activator of transcription 3; hENT1, human equilibrative nucleoside transporter 1; RRM, ribonucleoside diphosphate reductase.

exhibit an EMT phenotype, and GR-HPCCs exhibit a downregulated hENT1 expression level and upregulated RRM1 and RRM2 expression levels via TWIST1 and ZEB1 mediated by the HIF-1 $\alpha /$ STAT3 signaling pathway (Fig. 5D). More importantly, in xenografted pancreatic tumors induced by GR-Panc-1 cells, co-treatment with ASX and gemcitabine was able to significantly suppress tumor growth in comparison with treatment with gemcitabine alone. Furthermore, it was identified that co-treatment with ASX and gemcitabine suppressed tumor growth in the xenografted GR-Panc-1 model by inducing apoptosis.

Previous studies investigated that hENT1 was a key mediator of gemcitabine resistance in the clinic $(5,8)$. Additionally, RRM1 and RRM2, the targets of gemcitabine, were demonstrated to be associated with gemcitabine resistance (8,10-12). Although hENT1, RRM1 and RRM2 are known to associated with gemcitabine resistance, the underlying molecular mechanism remains unknown. It has been investigated previously that hENT1 was induced by peroxisome-proliferator-activated receptor (PPAR) $\alpha$ and PPAR $\gamma$ in GR-HPCCs (25), and RRM1 and RRM2 were mediated by the protein kinase $\mathrm{B}$, phosphoinositide 3-kinase, Ras/extracellular-signal-regulated kinase (ERK) and mitogen-activated protein kinase/ERK kinase 1/2 signaling pathways (26-30). The results of the present study indicated that TWIST1 and ZEB1, the transcription factors involved in EMT, are able to decrease hENT1 expression and increase RRM1 and RRM2 expression in GR-HPCCs treated with gemcitabine. Furthermore, ASX is able to reverse the effect of TWIST1 and ZEB1 to hENT1, RRM1 and RRM2 by inhibiting TWIST1 and ZEB1 expression. ASX was able to inhibit the EMT phenotype in GR-HPCCs treated with gemcitabine. Furthermore, HIF-1 $\alpha$ is 
A

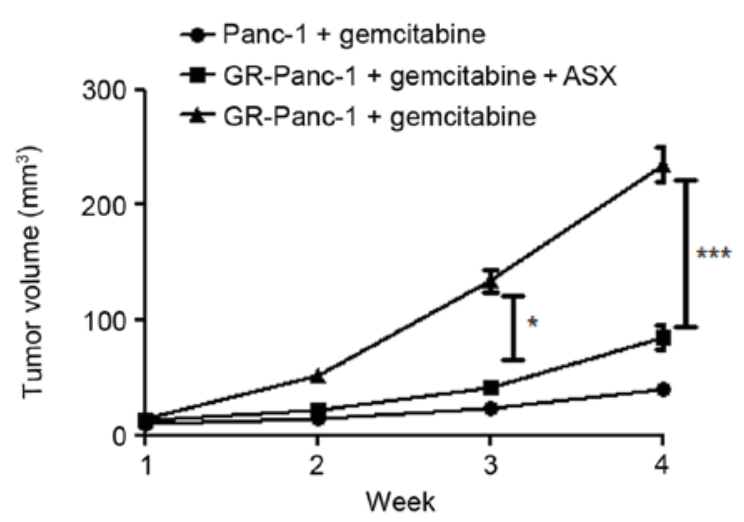

C

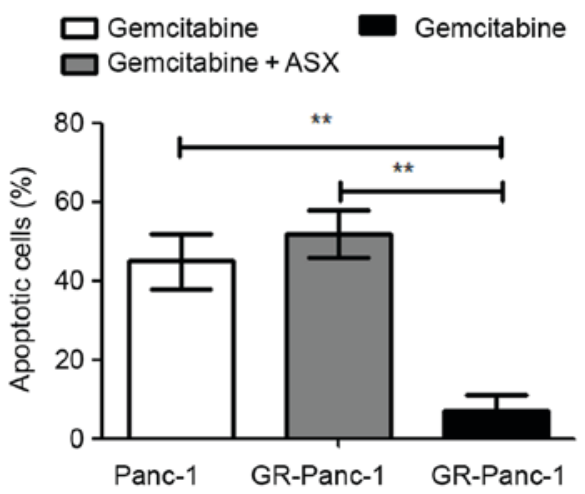

B

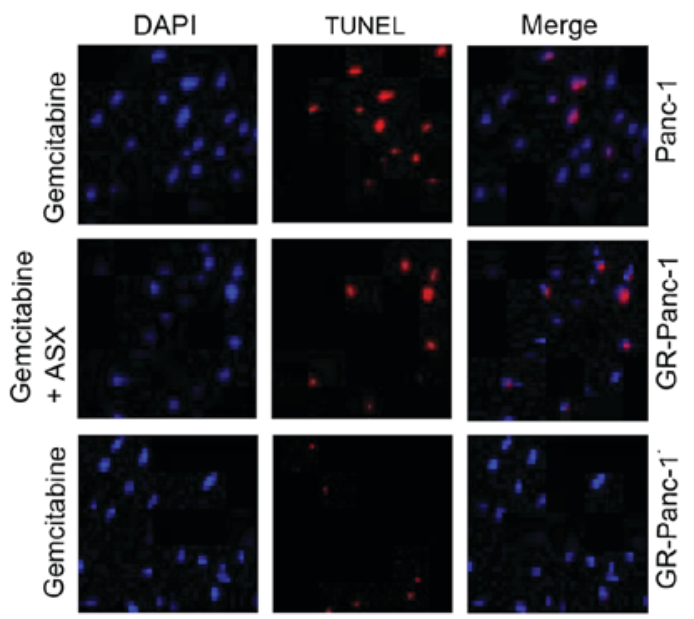

D

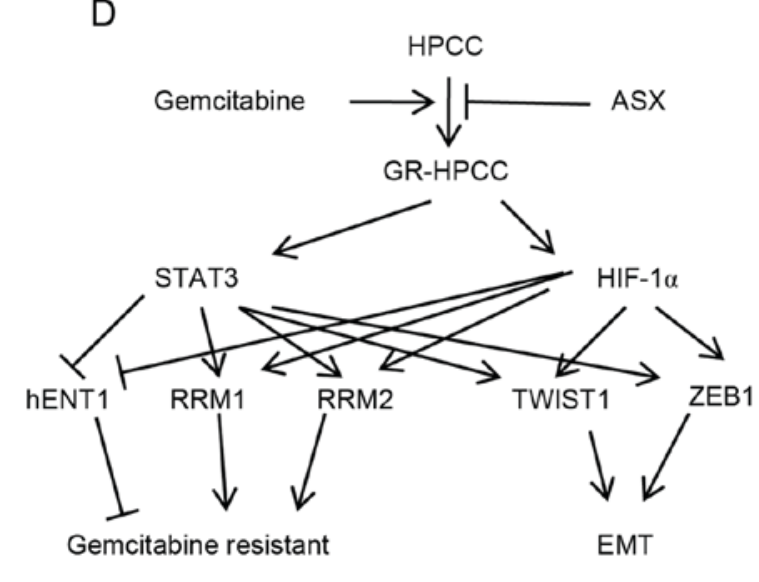

Figure 5. ASX inhibits the growth of gemcitabine-resistant pancreatic tumors in vivo through apoptosis. (A) Panc-1 or GR-Panc-1 cells were used to establish a xenograft transplantation model. At 2 days after inoculation, mice were treated with $10 \mathrm{mg} / \mathrm{kg}$ gemcitabine or co-treated with $500 \mathrm{mg} / \mathrm{kg}$ ASX ( $2 \mathrm{~h}$ before gemcitabine treatment) and $10 \mathrm{mg} / \mathrm{kg}$ gemcitabine 3 times/day through intraperitoneal injection. The tumor volume was measured weekly. (B) Apoptosis was determined using an In Situ Cell Death Detection kit. (C) Quantification of cell apoptosis. (D) Signaling pathway illustrating the effect of ASX inhibition on gemcitabine-resistant human pancreatic cancer progression through EMT inhibition and gemcitabine resensitization. ${ }^{*} \mathrm{P}<0.05 ;{ }^{* *} \mathrm{P}<0.001 ;{ }^{* * *} \mathrm{P}<0.005$. ASX, astaxanthin; HPCCs, human pancreatic cancer cells; GR-HPCCs, gemcitabine-resistant HPCCs; STAT3, signal transducer and activator of transcription 3; HIF-1 $\alpha$, hypoxia-inducible factor $1 \alpha$; hENT1, human equilibrative nucleoside transporter 1; RRM, ribonucleoside diphosphate reductase; EMT, epithelial-mesenchymal transition.

a nucleoprotein with transcriptional activity, which has a broad target gene spectrum, including hypoxia adaptation, tumor growth and inflammation-associated genes. When HIF-1 $\alpha$ binds to target genes, transcriptional and post-transcriptional regulation occurs and tumor growth may be accelerated (31). STAT3 is able to mediate growth factor receptors and signaling downstream of cytokines; activated STAT3 is able to promote aerobic glycolysis and downregulate mitochondrial activity, which may decrease reactive oxygen species (ROS) generation and delay senescence, and also promote survival and inhibit apoptosis in drug-resistant cells (32). In the present study, the participation of HIF-1 $\alpha$ and STAT3 in GR-HPCC mediation was investigated, together with the role of these factors in mediating the expression of TWIST1 and ZEB1, mediators of hENT1, RRM1 and RRM2 that contribute to EMT. Although HIF-1 $\alpha$ and STAT3 exhibited significant differences in expression between HPCCs and GR-HPCCs, ASX was able to reverse the alterations in GR-HPCCs treated with gemcitabine, and contribute to gemcitabine-induced cell death in GR-HPCCs.
Using a tumor xenograft model to evaluate the effect of ASX and gemcitabine co-treatment in vivo, it was identified that single administration of gemcitabine was able to suppress tumor growth in the gemcitabine-sensitive cell xenografted model, but not suppress tumor growth in the gemcitabine-resistance cell xenografted model; when co-treated with ASX and gemcitabine, tumor growth was significantly inhibited in the gemcitabine-resistance cell xenografted model, which supported the in vitro results. Furthermore, using in situ apoptosis detection, with ASX and gemcitabine co-treatment in the gemcitabine-sensitive cell xenografted model, the suppression of tumor growth occurred by enhancing apoptosis.

The results of the present study indicated that HIF-1 $\alpha /$ STAT3-TWIST1/ZEB1-EMT was a novel mechanism for gemcitabine resistance in GR-HPCCs. Furthermore, it is suggested that co-treatment with ASX and gemcitabine may be a novel and efficient therapeutic strategy for gemcitabine-resistant pancreatic cancer by targeting hENT1, RRM1 and RRM2, and inhibiting the gemcitabine-induced EMT phenotype. 


\section{References}

1. Siegel R, Naishadham D and Jemal A: Cancer statistics, 2013 CA Cancer J Clin 63: 11-30, 2013.

2. Diener MK, Combs SE and Büchler MW: Chemoradiotherapy for locally advanced pancreatic cancer. Lancet Oncol 14: 269-270, 2013

3. Akimoto M, Iizuka M, Kanematsu R, Yoshida M and Takenaga K Anticancer effect of ginger extract against pancreatic cancer cells mainly through reactive oxygen Species-mediated Autotic cell death. PLoS One 10: e0126605, 2015.

4. Seicean A, Petrusel L and Seicean R: New targeted therapies in pancreatic cancer. World J Gastroenterol 21: 6127-6145, 2015.

5. Nordh S, Ansari D and Andersson R: hENT1 expression is predictive of gemcitabine outcome in pancreatic cancer: A systematic review. World J Gastroenterol 20: 8482-8490, 2014

6. Poplin E, Wasan H, Rolfe L, Raponi M, Ikdahl T, Bondarenko I, Davidenko I, Bondar V, Garin A, Boeck S, et al: Randomized, multicenter, phase II study of CO-101 versus gemcitabine in patients with metastatic pancreatic ductal adenocarcinoma: Including a prospective evaluation of the role of hENT1 in gemcitabine or CO-101 sensitivity. J Clin Oncol 31: 4453-4461, 2013.

7. Wattanawongdon W, Hahnvajanawong C, Namwat $N$, Kanchanawat S, Boonmars T, Jearanaikoon P, Leelayuwat C, Techasen A and Seubwai W: Establishment and characterization of gemcitabine-resistant human cholangiocarcinoma cell lines with multidrug resistance and enhanced invasiveness. Int J Oncol 47: 398-410, 2015.

8. Jordheim LP and Dumontet C: Do hENT1 and RRM1 predict the clinical benefit of gemcitabine in pancreatic cancer? Biomark Med 7: 663-671, 2013

9. Yoneyama H, Takizawa-Hashimoto A, Takeuchi O, Watanabe Y, Atsuda K, Asanuma F, Yamada Y and Suzuki Y: Acquired resistance to gemcitabine and cross-resistance in human pancreatic cancer clones. Anticancer Drugs 26: 90-100, 2015.

10. Nakagawa N, Murakami Y, Uemura K, Sudo T, Hashimoto Y, Kondo N and Sueda T: Combined analysis of intratumoral human equilibrative nucleoside transporter 1 (hENT1) and ribonucleotide reductase regulatory subunit M1 (RRM1) expression is a powerful predictor of survival in patients with pancreatic carcinoma treated with adjuvant gemcitabine-based chemotherapy after operative resection. Surgery 153: 565-575, 2013.

11. Bhutia YD, Hung SW, Krentz M, Patel D, Lovin D, Manoharan R, Thomson JM and Govindarajan R: Differential processing of let-7a precursors influences RRM2 expression and chemosensitivity in pancreatic cancer: Role of LIN-28 and SET oncoprotein. PLoS One 8: e53436, 2013.

12. Fisher SB, Fisher KE, Patel SH,Lim MG, Kooby DA,El-Rayes BF, Staley CA III, Adsay NV, Farris AB III and Maithel SK: Excision repair cross-complementing gene-1, ribonucleotide reductase subunit M1, ribonucleotide reductase subunit M2, and human equilibrative nucleoside transporter-1 expression and prognostic value in biliary tract malignancy. Cancer 119: 454-462, 2013

13. Namba T, Kodama R, Moritomo S, Hoshino T and Mizushima T: Zidovudine, an anti-viral drug, resensitizes gemcitabine-resistant pancreatic cancer cells to gemcitabine by inhibition of the Akt-GSK3ß-Snail pathway. Cell Death Dis 6: e1795, 2015.

14. Yi XP,Han T, Li YX, Long XY and Li WZ: Simultaneous silencing of XIAP and survivin causes partial mesenchymal-epithelial transition of human pancreatic cancer cells via the PTEN/PI3K/Akt pathway. Mol Med Rep 12: 601-608, 2015

15. Cheng ZX, Wang DW, Liu T, Liu WX, Xia WB, Xu J, Zhang YH, Qu YK, Guo LQ, Ding L, et al: Effects of the HIF-1 $\alpha$ and NF- $\kappa$ B loop on epithelial-mesenchymal transition and chemoresistance induced by hypoxia in pancreatic cancer cells. Oncol Rep 31: 1891-1898, 2014

16. Rao AR, Sindhuja HN, Dharmesh SM, Sankar KU, Sarada R and Ravishankar GA: Effective inhibition of skin cancer, tyrosinase, and antioxidative properties by astaxanthin and astaxanthin esters from the green alga Haematococcus pluvialis. J Agric Food Chem 61: 3842-3851, 2013
17. Nakao R, Nelson OL, Park JS, Mathison BD, Thompson PA and Chew BP: Effect of dietary astaxanthin at different stages of mammary tumor initiation in BALB/c mice. Anticancer Res 30: 2171-2175, 2010.

18. Vena F, Li Causi E, Rodriguez-Justo M, Goodstal S, Hagemann T, Hartley JA and Hochhauser D: The MEK1/2 inhibitor pimasertib enhances gemcitabine efficacy in pancreatic cancer models by altering ribonucleotide reductase subunit-1 (RRM1). Clin Cancer Res 21: 5563-5577, 2015

19. Lai IL, Chou CC, Lai PT, Fang CS, Shirley LA, Yan R, Mo X, Bloomston M, Kulp SK, Bekaii-Saab T and Chen CS: Targeting the Warburg effect with a novel glucose transporter inhibitor to overcome gemcitabine resistance in pancreatic cancer cells. Carcinogenesis 35: 2203-2213, 2014

20. D'Angelo RC, Liu XW, Najy AJ, Jung YS, Won J, Chai KX, Fridman R and Kim HR: TIMP-1 via TWIST1 induces EMT phenotypes in human breast epithelial cells. Mol Cancer Res 12: 1324-1233, 2014

21. Díaz-López A, Díaz-Martín J, Moreno-Bueno G, Cuevas EP, Santos V, Olmeda D, Portillo F, Palacios J and Cano A: Zeb1 and Snail 1 engage miR-200f transcriptional and epigenetic regulation during EMT. Int J Cancer 136: E62-E73, 2015.

22. Cho KH, Choi MJ, Jeong KJ, Kim JJ, Hwang MH, Shin SC, Park CG and Lee HY: A ROS/STAT3/HIF-1 $\alpha$ signaling cascade mediates EGF-induced TWIST1 expression and prostate cancer cell invasion. Prostate 74: 528-536, 2014.

23. Zhang W, Shi X, Peng Y, Wu M, Zhang P, Xie R, Wu Y, Yan Q, Liu S and Wang J: HIF-1 $\alpha$ promotes epithelial-mesenchymal transition and metastasis through direct regulation of ZEB1 in colorectal cancer. PLoS One 10: e0129603, 2015.

24. Xiong H, Hong J, Du W, Lin YW, Ren LL, Wang YC, Su WY, Wang JL, Cui Y, Wang ZH and Fang JY: Roles of STAT3 and ZEB1 proteins in E-cadherin down-regulation and human colorectal cancer epithelial-mesenchymal transition. J Biol Chem 287: 5819-5832, 2012.

25. Montero TD, Racordon D, Bravo L, Owen GI, Bronfman ML and Leisewitz AV: PPAR $\alpha$ and PPAR $\gamma$ regulate the nucleoside transporter hENT1. Biochem Biophys Res Commun 419: 405-411, 2012.

26. Lei W, Feng XH, Deng WB, Ni H, Zhang ZR, Jia B, Yang XL, Wang TS, Liu JL, Su RW, et al: Progesterone and DNA damage encourage uterine cell proliferation and decidualization through up-regulating ribonucleotide reductase 2 expression during early pregnancy in mice. J Biol Chem 287: 15174-15192, 2012.

27. Kaira $\mathrm{K}$ and Yamamoto N: Prognostic and predictive factors in resected non-small-cell lung cancer. Expert Opin Med Diagn 4: 373-381, 2010.

28. Tassone P, Di Martino MT, Ventura M, Pietragalla A, Cucinotto I, Calimeri T, Bulotta A, Neri P, Caraglia M and Tagliaferri P: Loss of BRCA1 function increases the antitumor activity of cisplatin against human breast cancer xenografts in vivo. Cancer Biol Ther 8: 648-653, 2009.

29. El-Khoueiry AB, Ramanathan RK, Yang DY, Zhang W, Shibata S, Wright JJ, Gandara D and Lenz HJ: A randomized phase II of gemcitabine and sorafenib versus sorafenib alone in patients with metastatic pancreatic cancer. Invest New Drugs 30: 1175-1183, 2012.

30. Kao YT, Hsu WC, Hu HT, Hsu SH, Lin CS, Chiu CC, $\mathrm{Lu} \mathrm{CY}$, Hour TC, Pu YS and Huang AM: Involvement of p38 mitogen-activated protein kinase in acquired gemcitabine-resistant human urothelial carcinoma sublines. Kaohsiung J Med Sci 30: 323-330, 2014

31. Yan Q, Chen P, Wang S, Liu N, Zhao P and Gu A: Association between HIF-1 $\alpha$ C1772T/G1790A polymorphisms and cancer susceptibility: An updated systematic review and meta-analysis based on 40 case-control studies. BMC Cancer 14: 950, 2014

32. Huang $\mathrm{C}$ and Xie K: Crosstalk of Sp1 and Stat 3 signaling in pancreatic cancer pathogenesis. Cytokine Growth Factor Rev 23 25-35, 2012. 